\title{
Atom trap trace analysis of Ca-41 samples at 1E-15 abundance level
}

TONG-YAN XIA, WEI-WEI SUN, SVEN EBSER, WEI JIANG, TIAN XIA AND ZHENG-TIAN LU

University of Science and Technology of China

Presenting Author: xty2012@mail.ustc.edu.cn

Calcium is an abundant element in the Earth's crust and minerals. It also exists extensively in organisms, especially in bones, teeth and shells. Research on the long-lived radioactive Ca-41 $\left(\mathrm{t}_{1 / 2}=9.94 \times 10^{5} \mathrm{yr},{ }^{41} \mathrm{Ca} / \mathrm{Ca}=2 \times 10^{-15} \sim 3 \times 10^{-14}\right)$ contributes to investgating kinetic processes in geoscience and biomedical science. Besides, G. M. Raisbeck and F. Yiou have proposed the possibility of using Ca-41 for radioactive dating in 1979 [1]. Ca41 is a candidate for dating samples ranging from $5 \times 10^{4}$ to $1 \times 10^{6}$ years of age, expected to cover the gap between the dating ranges of C-14 and Cl-36.

Accelerator Mass Spectrometry (AMS) is the most common and sensitive method to analyze $\mathrm{Ca}-41$ samples at present. A ${ }^{41} \mathrm{Ca} / \mathrm{Ca}=(1.9 \pm 0.5) \times 10^{-15}$ value has been measured by Wallner $e t$ al in 2004 [2]. For samples with Ca-41 abundance below $1 \times 10^{-13}$, the usage of $\mathrm{CaH}_{2}$ (to generate molecule ion $\mathrm{CaH}_{3}^{-}$) is necessary, which must be under careful treatment during production and storage, and the terminal voltage of Accelerator must be raised over $3 \mathrm{MV}$, to eliminate the interference of K-41 - the isobar and decay product of $\mathrm{Ca}-41$. Consequently, only several AMS systems can reach this sensitivity.

Atom trap trace analysis (ATTA) is an atomic optics technique for rare isotopes trace measurement [3]. It has been successfully applied on radioactive $\mathrm{Kr}-81, \mathrm{Kr}-85$ and $\mathrm{Ar}-39$ dating. With our Ca-ATTA apparatus, it takes 10 hours to analyze a ${ }^{41} \mathrm{Ca} / \mathrm{Ca}=1 \times 10^{-15}$ sample with statistical uncertainty of $10 \%$, consuming $160 \mathrm{mg}$ Ca. The metallic calcium samples are prepared by reducing $\mathrm{CaO}$ with aluminum from $\mathrm{CaCO}_{3}$, produced from $\mathrm{Ca}^{2+}$ solutions through $\mathrm{CaC}_{2} \mathrm{O}_{4}$, with yield of $55 \%$.

In an ATTA apparatus, atoms of interest can be selectively cooled and trap by laser resonant to their characteristic electron transitions in magnetic fields. This operation isolates all other elements or isotopes by laser frequency tuning. The atoms in the trap are detected by measuring fluorescence with sensitivity of single atom counting.

[1]M. Raisbeck \& F. Yiou (1979). Nature 277(5691), 42-44.

[2]Wallner, A. , et al (2004). Nuclear Inst \& Methods in Physics Research B 223, 759-764.

[3]Chen, Y. ,el al. Science 286(5442), 1139-1141. 